\title{
Les résidus de pesticides organochlorés dans les laits animaux et humains
}

\author{
par \\ F. M. LUQUET*, J. GOURSAUD** et J. CASALIS*** \\ ENSIA, Douai
}

Pendant des millénaires l'homme n'a vécu que des produits de la cueillette et de la chasse : plantes et animaux sauvages qui permettaient d'assurer sa subsistance.

Devenu sédentaire, il a cultivé le sol, domestiqué les végétaux et les animaux.

Aujourd'hui dans les pays développés, seule une faible partie de la population suffit à produire les quantités de denrées alimentaires nécessaires à l'alimentation de l'ensemble des habitants, et ceci grâce aux perfectionnements des techniques agronomiques, en particulier en ce qui concerne la sélection végétale et animale, la fertilisation et l'irrigation des sols, la mécanisation de l'agriculture et les moyens de lutte contre les maladies et parasites des plantes et des animaux.

Parmi ces moyens de lutte les pesticides occupent actuellement une place prépondérante : C'est ainsi que l'on a utilisé en France en $196719000 \mathrm{t}$ et produit aux Etats-Unis en $1969550000 \mathrm{t}$ de matières actives entrant dans leur composition.

On ne peut, dans l'état actuel de nos connaissances se passer de ces produits. Ils permettent d'éviter une réduction considérable des rendements agricoles qui entraînerait à brève échéance même dans les pays d'agriculture intensive la réapparition de la disette sinon de la famine. Mais ils ont un grave inconvénient : Ce sont des substances toxiques et certains pesticides peuvent laisser dans les denrées alimentaires des résidus dont on ne connaît pas de façon précise les effets que leur ingestion répétée peut avoir sur la santé de 1 'homme.

\footnotetext{
* Extrait de thèse de Doctorat d'Université, mention Sciences (avril 1973).

** INRA Chaire d'industrie laitière

*** Professeur d'Industrie Laitière.
} 


\section{GENERALITES}

\section{I. - LES PESTICIDES}

Pesticide est un dérivé du mot anglais " pest " signifiant fléau, qui est actuellement employé pour désigner les produits antiparasitaires.

Les pesticides peuvent être groupés :

- Soit en fonction de leur utilisation : herbicides, fongicides, insecticides pour les trois classes principales ; bactéricides, acaricides, nématicides, etc.

- Soit en fonction de la matière active qu'ils contiennent.

En France environ 300 matières actives différentes dont la vente est autorisée entrent dans la fabrication de 4000 spécialités commerciales.

Les matières actives sont des substances chimiques simples ou complexes, minérales ou organiques, naturelles ou de synthèse.

Les principaux groupes de matières actives sont les suivants :

a) Groupe des urées substituées. Ce sont essentiellement des herbicides, qui inhibent la photosynthèse des feuilles.

b) Groupe des triazines. Ces produits herbicides inhibent la division cellulaire des plantes.

c) Groupe des carbamates. Ces substances dérivées de l'acide carbamique sont employées comme herbicides, fongicides, ou insecticides.

d) Groupe des dérivés du benzène. Ce groupe chimique est très important en phytopharmacie, il comprend des insecticides dérivés des phénols, crésols ou colorants nitrés, et des fongicides.

e) Groupe des organophosphorés. Ces composés sont caractérisés par leur molécule qui dérive toujours de l'un des acides phosphoriques ou thiophosphoriques. Ce sont essentiellement des esters ou des amides des acides contenant du phosphore. Leur propriété toxique commune est l'inhibition des cholinestérases.

f) Groupe des organohalogénés. Ce sont des substances dérivées de noyaux aromatiques dans lesquels on ne trouve que des atomes de carbone, d'hydrogène et de chlore. Ce sont essentiellement des insecticides agissant par contact ou ingestion. Leurs principales propriétés communes sont une très grande stabilité, une grande rémanence, une très faible solubilité dans l'eau, ainsi qu'une forte solubilité dans les matières grasses.

Leur liposolubilité est la cause de l'accumulation des pesticides chlorés très rémanents dans les graisses végétales, animales ou humaines. 
Le lait et les produits laitiers qui contiennent une proportion importante de lipides, sont donc particulièrement exposés à contenir des traces de résidus d'insecticides chlorés.

\section{II. - LES PESTICIDES ORGANOCHLORES}

\section{1) Les pesticides organochlorés peuvent être divisés en quatre groupes principaux}

a) Groupe $d u H C H$ (hexachlorocyclohexane : $\mathrm{C}_{6} \mathrm{H}_{6} \mathrm{Cl}_{6}$ ), ne comprenant qu'un seul cycle benzénique, mais avec plusieurs isomères optiques, dont les principaux sont : $\mathrm{HCH} \alpha, \mathrm{HCH} \beta, \mathrm{HCH} \gamma$.

Le $\mathrm{HCH}$ technique couramment employé contenait :

$$
\begin{array}{r}
60-70 \text { p. } 100 \text { de } \alpha \\
5-12 \text { p. } 100 \text { de } \beta \\
10-15 \text { p. } 100 \text { de } \gamma
\end{array}
$$

Le lindane, seul isomère ayant des propriétés insecticides intéressantes, contient 99 p. 100 de $\mathrm{HCH} \gamma$ (fig. 1).<smiles>ClC(Cl)(Cl)C(Cl)(Cl)Cl</smiles>
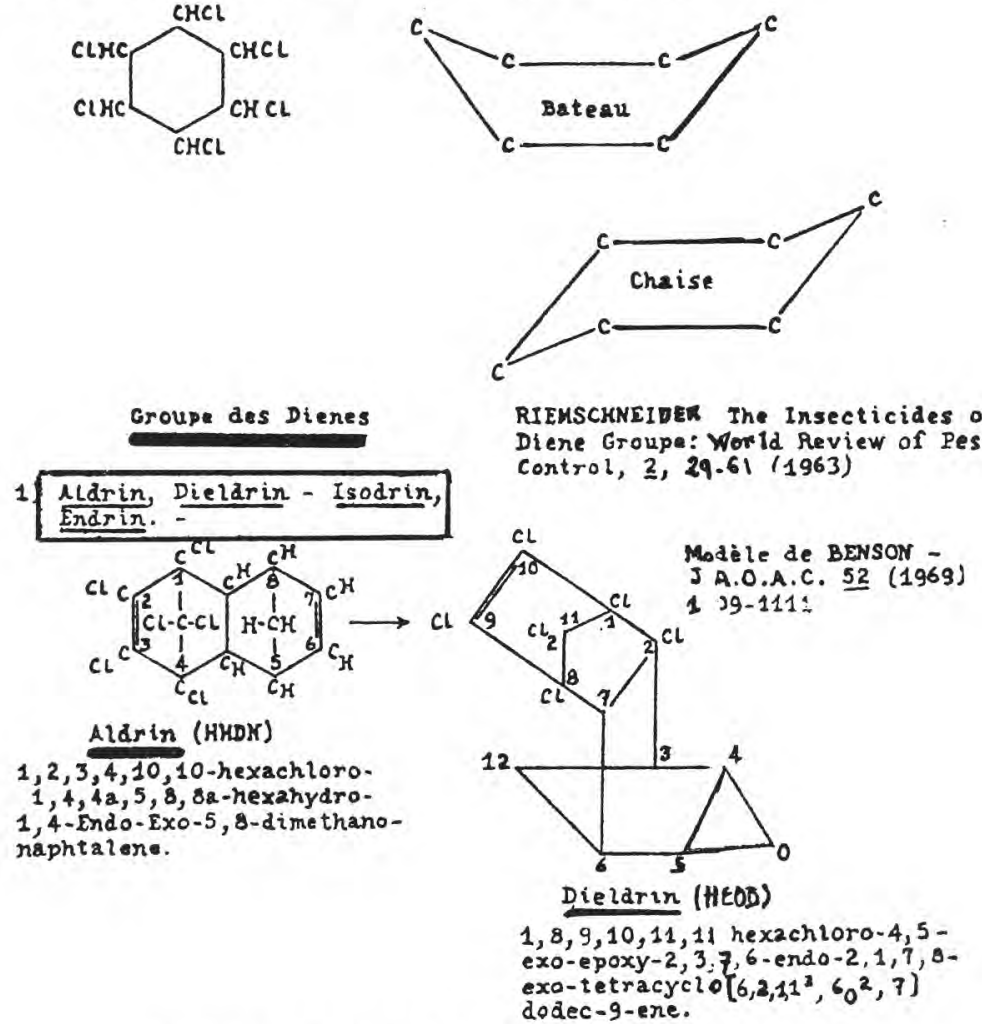

fig. 1 d'après E. L. Delvaux (J. Pharm. Belg., 1971)

Groupe du cyclohexane chloré

Le $\mathrm{HCH}$ est un mélange non constant d'au moins 4 isomères d'addition au benzène : soit le $\alpha, \beta, \gamma, \delta$ hexachlorocyclohexane ; seul l'isomère $\gamma$ en proportion de \pm 12 p. 100 (lindane ou gammexane), est actif.

La molécule du hexachlorocyclohexane pourrait exister sous 16 formes stéréosimériques du fait des positions respectives de « $\mathrm{H}$ » et « $\mathrm{Cl}$ » et de la forme chaise et bateau. 
b) Groupe du $D D T$ (dichlorodiphényltrichloréthane : $\mathrm{C}_{14} \mathrm{H}_{\imath} \mathrm{Cl}_{5}$ ). Comportant deux cycles benzéniques reliés par une chaîne éthane. Ce produit est souvent considéré comme synonyme d'organochloré, car il est parmi les insecticides celui dont la renommée est la plus grande en raison de sa très forte utilisation depuis 1945. Dans le même groupe figurent des composés métabolites du DDT, le DDD (dichlorodiphényldichloroéthane) et le DDE (dichlorodiphényldichloroéthylène) (fig. 2).

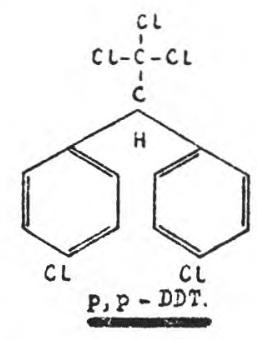

1,1,1-Trichloro-2,2-bis(p-chloropheny l) ethare

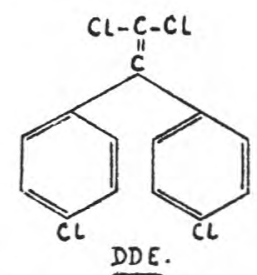

1,1-dichloro-2,2-bis(p-chloro phenyl) ethene.

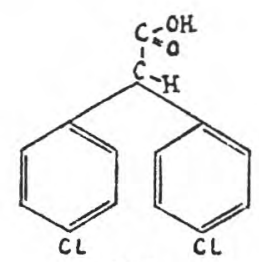

DDA.

2, 2-bis(p-chlorophenyl) 2cetic acid

Le DDT technique est un mélange de $0, p$ et $p, p-D D F$, de DDE et IDE - impuretés.

DDMU = Dichlordiphenylmonounsaturated.

fig. 2 d'après E. L. Delvaux (J. Pharm. Belg., 1971)

Groupe du DDT

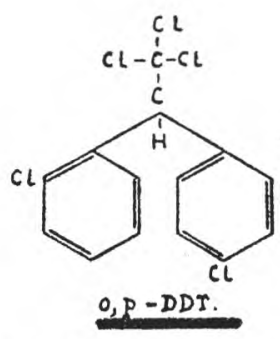

1,1,1,-Trichloro-2-(0-chlorophe ryl-2-(p-chlorophenyl) ethane.<smiles>ClC(Cl)(Cl)c1ccccc1</smiles>

TDE ou DDD Rothane (I)

1,1-dichloro-2,2-bis(p-chloropheryl) ethare.

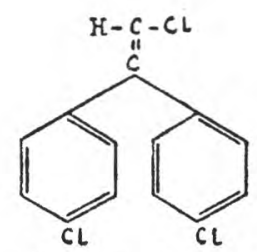

DDMU Ou TDE olefine

1-cr.loro-2,2-bis(p-chlorophanyl) ethene 
c) Groupe du chlordane constitué par un assemblage complexe d'un noyau benzénique et indène (chlordane, heptachlore et heptachlore époxyde) ou par deux noyaux benzéniques accolés (aldrine, dieldrine) (fig. 1 et 3 ).
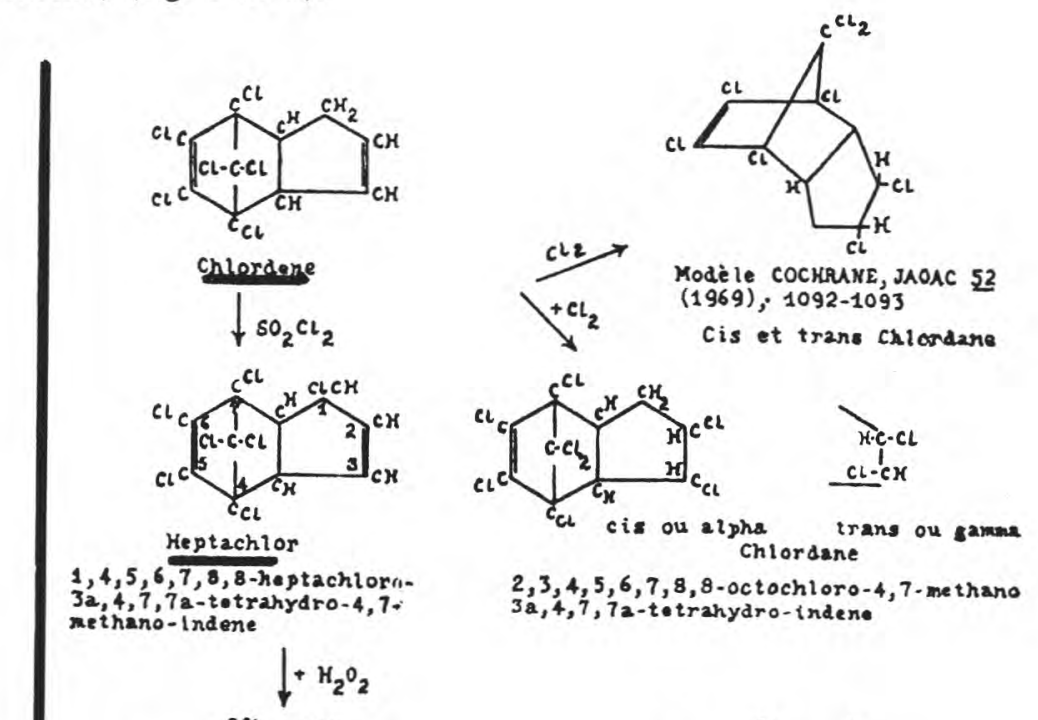

$$
\begin{aligned}
& 2,3,4,5,6,7,8,8-o c t o c h l o r o-4,7 \text { - we thano } \\
& 3 \mathrm{a}, 4,7,7 \mathrm{a}-\mathrm{t} \text { tranydro-indene }
\end{aligned}
$$
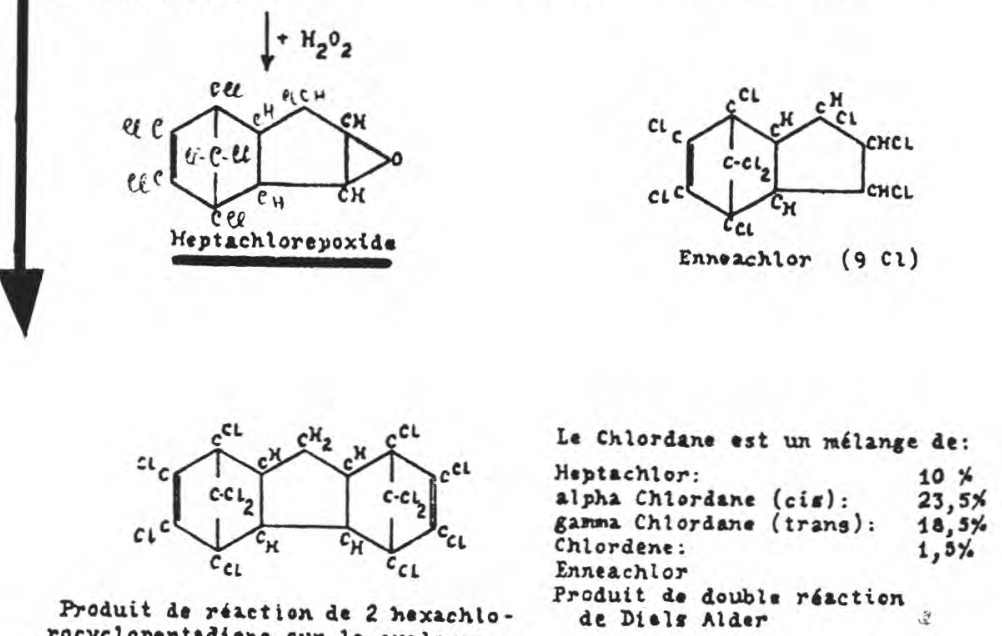

de Diels Alder

Produit de reaction de 2 hexachlorocyclopentadiene sur 2 eyclopen -

radiene

fig. 3 d'après E. L. Delvaux (J. Pharm. Belg., 1971)

Chlordane, heptachlore

d) Dérivés de l'essence de térébenthine, ou camphènes chlorés.

Ces dérivés sont très stables et peuvent persister dans le sol plusieurs années. Ils peuvent cependant se dégrader ou se métaboliser sous l'action de facteurs chimiques, physiques ou biochimiques. La métabolisation la plus fréquente est l'époxydation qui conduit souvent à un composé plus stable et surtout plus toxique que la substance de départ (aldrine $\stackrel{\text { époxydation }}{\longrightarrow}$ dieldrine). 


\section{2) Comportement des pesticides organochlorés dans les milieux habituels d'emploi}

a) $H C H$. Il était utilisé en particulier à l'état technique comme insecticide dans les badigeons de blanchiment des étables. La persistance de ce produit est variable selon les isomères qui disparaissent par volatilisation, le plus stable et rémanent étant le $\beta \mathrm{HCH}$, le plus volatil étant le $\gamma \mathrm{HCH}$.

b) $D D T$. Ce produit se métabolise lentement et en particulier selon le schéma :

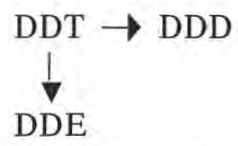

c) Heptachlore et aldrine. Dans le sol ou dans les eaux, par époxydation, ces deux produits donnent respectivement l'heptachlore époxyde et la dieldrine qui constituent la forme stable des produits initiaux.

\section{3) Les effets de la technologie sur la teneur des produits laitiers en résidus de pesticides}

Les organochlorés étant liposolubles, se rassemblent au cours des processus technologiques dans la matière grasse des produits fabriqués. Ainsi, à partir du lait, on obtient par écrémage, suivi de barratage, le beurre dans lequel se retrouvent pratiquement tous les pesticides chlorés du lait et le lait écrémé qui en est pratiquement exempt.

Les procédés technologiques couramment employés en industrie laitière, ont des effets variables sur la dégradation des pesticides chlorés. C'est ainsi que des diminutions de 50 p. 100 du taux initial peuvent être observées lors du traitement du lait par déshydratation (fig. 4).

\section{4) Toxicologie des pesticides organochlorés et intoxications}

a) Chez l'homme. La pénétration des pesticides dans l'organisme humain se fait le plus souvent par voie pulmonaire ou par voie percutanée avec une intensité variable selon le solvant employé. Ce sont ces deux modes d'absorption qui sont le plus souvent la cause d'intoxications aiguës non volontaires. La pénétration par voie digestive peut conduire à de graves intoxications aiguës dans le cas d'une absorption consciente de pesticides. Par contre l'ingestion répétée de petites doses de résidus (par les aliments en particulier), ne provoque pas immédiatement de troubles graves, mais on en connaît très mal les conséquences éventuelles à long terme.

La revue "Gigiena $i$ sanitarija " donne une statistique portant sur 68 pays et concernant les substances les plus souvent mises en cause lors d'intoxications (tab. 1). 


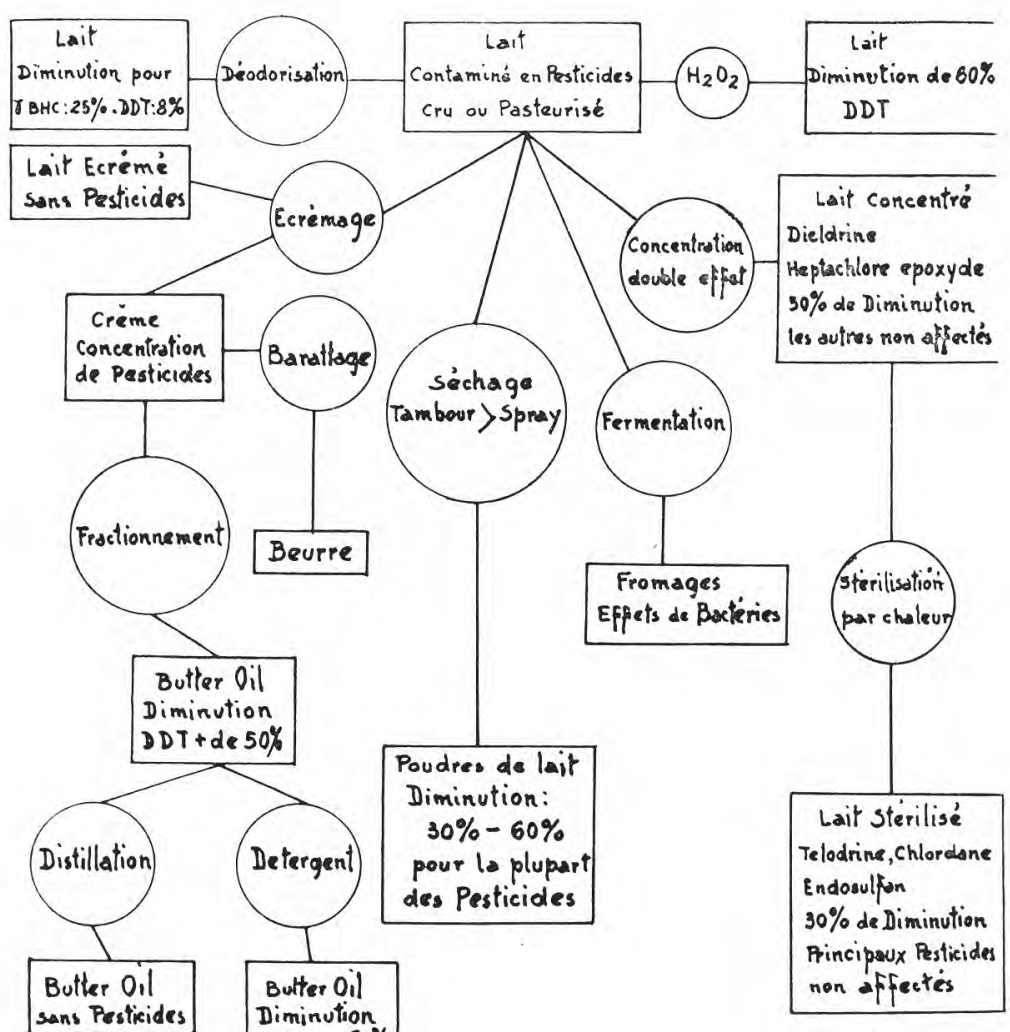

fig. 4

Pesticides et technologie laitière

TABLEAU 1

\begin{tabular}{l|c}
\hline Substances toxiques & Fréquence sur 100 intoxications \\
\cline { 2 - 2 } Organophosphorés & 30,3 \\
Organochlorés & 41,1 \\
Arsénicaux & 19,7 \\
Organomercuriques & 7,9 \\
Autres & 3 \\
\hline
\end{tabular}

En intoxication aiguë les résidus de pesticides organochlorés atteignent surtout les organes parenchymateux, hématopoiétiques, nerveux ; les systèmes cardiaques ou gastro-intestinaux sont moins affectés.

De nombreux auteurs ont montré que le taux d'accumulation des pesticides chez les femmes enceintes, est moindre que chez celles 
qui ne le sont pas. Ceci pourrait s'expliquer par un métabolisme lipidique plus ou moins actif pendant la grossesse.

Par contre le placenta et les tissus d'enfants morts-nés, contiennent des quantités assez importantes de résidus de pesticides organochlorés.

Les nourrissons dont le système nerveux paraît particulièrement sensible aux effets des pesticides et spécialement du DDT, ne sont pas protégés du risque de toxicité pouvant résulter de l'ingestion de lait et spécialement de lait humain.

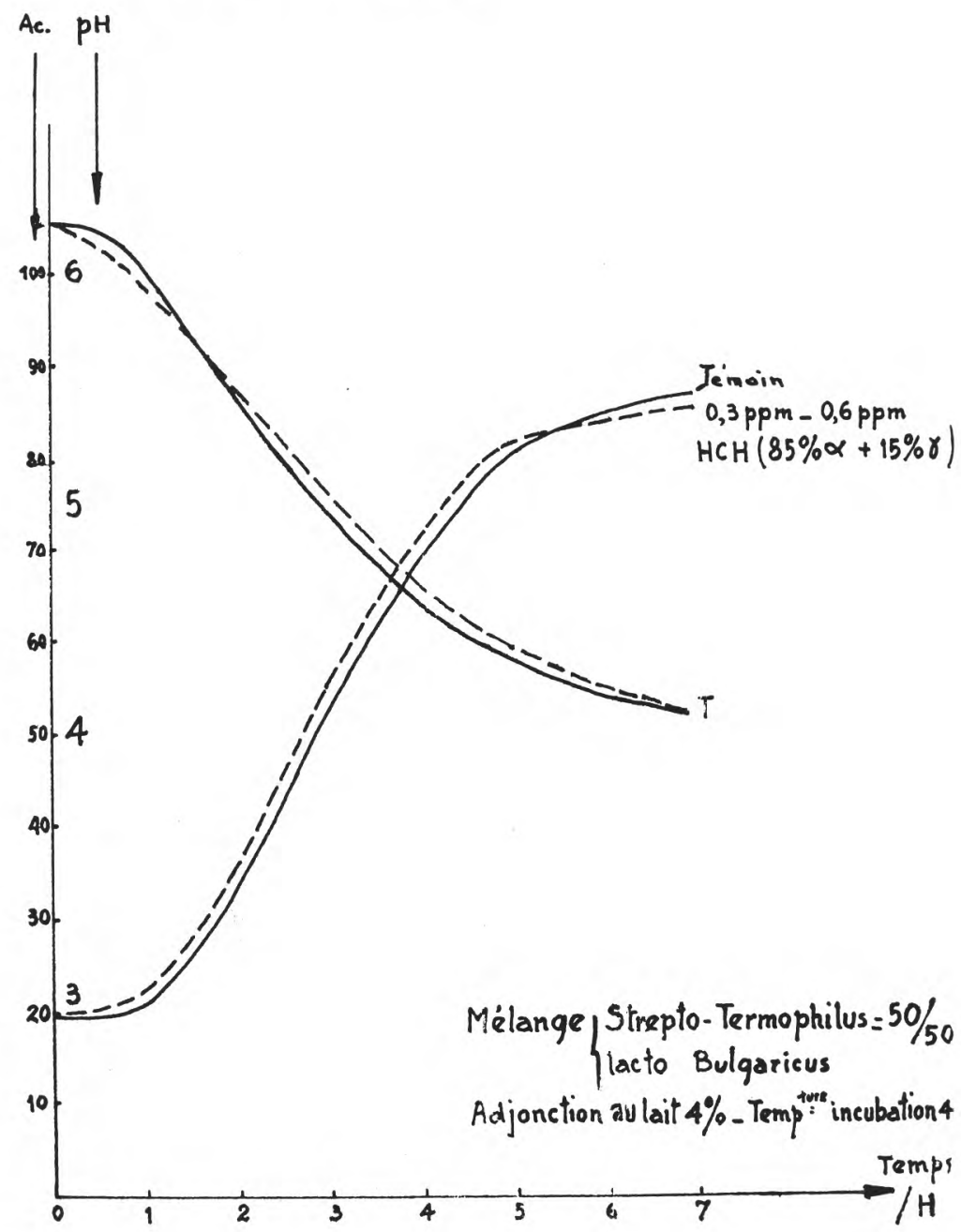

fig. 5 
De plus comme l'a montré Truhaut, certains pesticides sont susceptibles de créer des phénomènes de cancérisation.

b) Chez l'animal. Parmi les cas d'intoxication des animaux par les résidus de pesticides, il faut souligner la possibilité de nocivité du lait de la vache pour son veau. Ce dernier pourra présenter des troubles nerveux ou des convulsions, lorsque celle-ci consomme du fourrage souillé par des traces de DDT, qui est sans effet sur la mère.

c) Sur les fermentations. Les travaux relatifs à l'action des résidus de pesticides organochlorés sur la flore des aliments crus ou transformés sont peu importants. D'après nos études, aucune action notable n'a pu être observée sur la fermentation lactique lors de la fabrication du yaourt, sous l'effet de lindane ou de $\mathrm{HCH} \alpha$ employé à la dose maximum de 0,6 ppm (fig. 5).

\section{III. - LEGISLATION}

En France, il existe depuis 1947 une législation, s'adaptant au cours des années à l'évolution de l'industrie des produits phytosanitaires. Cette réglementation vise toujours à protéger les plantes, aussi bien que les animaux et les hommes contre tout risque de nocivité pouvant résulter de l'emploi généralisé dans de nombreux secteurs économiques de pesticides.

Un des derniers textes législatifs est constitué par l'arrêté du 2 octobre 1972 qui interdit tout emploi en agriculture des pesticides suivants : aldrine, dieldrine, heptachlore et chlordane.

D'autre part, en ce qui concerne la pollution éventuelle des aliments par des résidus de substances utilisées en agriculture et en élevage, en raison des dangers que de telles substances pourraient occasionner à la santé humaine, un décret du 30 juillet 1971 interdit de détenir, de mettre en vente ou de vendre de telles denrées ou boissons.

Dans la pratique, cette dernière mesure se traduit par la fixation par chaque pays de normes de tolérances applicables à toutes les denrées alimentaires. Ainsi sont établis des seuils limites en résidus de pesticides par exemple, qui lorsqu'ils sont dépassés, rendent la marchandise non conforme, et placent le fabricant en état d'infraction.

Les tolérances administratives qui sont actuellement appliquées par de nombreux pays, ont été mises au point avec soin par les experts de l'Organisation Mondiale de la Santé, en tenant compte de la toxicité à long terme pouvant résulter de l'ingestion quotidienne de doses infimes de pesticides (tab. 2).

\section{IV. - METHODES DE RECHERCHE}

Le dosage des résidus de pesticides est lié au fait que ces produits sont présents à des doses infinitésimales dans des substrats très variés. Les traces de ces éléments se dosent en partie par million, c'est-à-dire en $\mathrm{mg}$ de substance novice par $\mathrm{kg}$ de denrée. 
TABLEAU 2

Tolérances administratives en résidus de pesticides organochlorés dans le lait et les produits laitiers

\begin{tabular}{|c|c|c|c|c|c|}
\hline Pesticides organochlorés & U.S.A. & Canada & Suisse & Allemagne & Codex OMS/FAO \\
\hline $\mathrm{HCB}$ & 0,30 & 0,30 & 0,30 & 0,50 & 0,30 \\
\hline $\begin{array}{c}\mathrm{HCH} \alpha+\beta+\delta \\
\beta \\
\gamma \text { (lindane) } \\
\text { Total } \\
\alpha+\beta+\gamma+\delta\end{array}$ & 0,30 & $\begin{array}{l}0,30 \\
0,30 \\
0,30\end{array}$ & $\begin{array}{l}0,10 \\
0,20\end{array}$ & $\begin{array}{l}0,10 \\
0,20\end{array}$ & 0,20 \\
\hline Heptachlore + heptachlore époxyde & 0,30 & 0,30 & 0,125 & 0,15 & 0,125 \\
\hline Aldrine + dieldrine & 0,30 & 0,10 & 0,125 & 0,15 & 0,125 \\
\hline $\mathrm{DDT}+\mathrm{DDD}+\mathrm{DDE}$ & 1,25 & 1,00 & 0,125 & 1,00 & 1,25 \\
\hline Chlordane & & 0,10 & & 0,05 & \\
\hline Endrine & & & & 0,02 & \\
\hline
\end{tabular}


Les déterminations qualitatives et quantitatives de ces résidus ont nécessité l'utilisation de nouvelles techniques d'extraction et de dosage analytique. Généralement les opérations de dosage comprennent quatre étapes principales :

- échantillonnage,

- extraction,

- enrichissement et purification,

- dosages analytiques.

\section{1) Echantillonnage}

L'échantillonnage constitue une phase importante du dosage, puisque cette fraction devra être représentative du produit global en qualité et quantité.

Dans le contexte de notre étude, chaque échantillon a été prélevé selon les directives de la norme FIL-IDF no 21958 (tab. 3).

TABLEAU 3

\begin{tabular}{l|l}
\hline \multicolumn{1}{c|}{$\begin{array}{c}\text { Nature du produit } \\
\text { - Lait }\end{array}$} & $\begin{array}{l}\text { Volume et emballage } \\
250 \mathrm{ml} \text { en flacon de verre à bouchon émeri. } \\
\text { Récipient rincé à l'hexane. }\end{array}$ \\
\hline - Fromage à la pièce et beurre & $\begin{array}{l}\text { Emballage commercial par unité de vente } \\
(250 \mathrm{~g}) .\end{array}$ \\
\hline $\begin{array}{l}\text { - Fromage de gros volume } \\
\text { (Cantal, Emmental) }\end{array}$ & $\begin{array}{l}250 \mathrm{~g} \text { prélevés avec une sonde inox en 10 } \\
\text { endroits différents, emballage de papier alu- } \\
\text { minium. }\end{array}$ \\
\hline
\end{tabular}

\section{2) Méthodes d'extraction}

De façon générale, ces méthodes employées ne doivent pas altérer la structure des pesticides ; et à cet égard, il est recommandé d'éviter les fortes températures et les milieux acides ou basiques.

a) Macération ou broyage. Ces techniques conviennent aux substrats solides et permettent une bonne homogénéisation (tourteaux, fromages, laits secs).

La qualité des solvants d'extraction est essentielle et leur pureté doit être vérifiée avant usage.

Nous avons retenu l'éther de pétrole, non miscible à l'eau pour les pesticides lipophiles et en particulier pour les organochlorés. 
L'utilisation d'un solvant polaire, miscible à l'eau, tel que l'acétonitrile ou l'acétone, permet un contact plus intime avec les substrats humides.

b) Extraction au soxhlet. Cette méthode s'applique aux échantillons secs. Cependant il est nécessaire de surveiller la température qui peut être la cause d'une dégradation partielle de certains pesticides.

\section{3) Enrichissement et purification}

Les méthodes d'extraction permettent d'obtenir des solutions très diluées en pesticides ainsi qu'en certaines autres substances

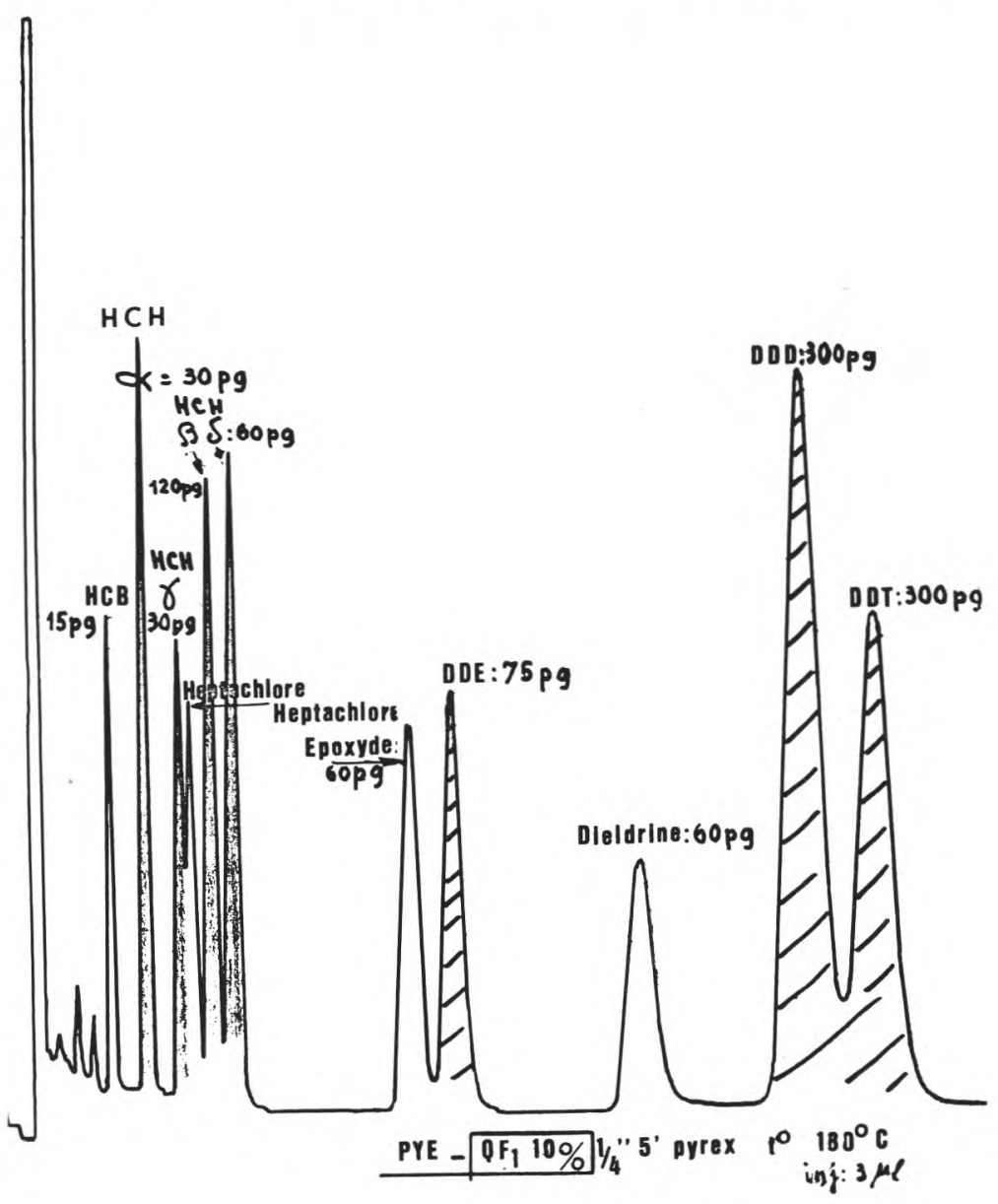

fig. 6

(chromatogramme 1) 
coextraites. Ces solutions sont généralement concentrées par évaporation du solvant à faible température et sous pression réduite.

Pour purifier les solutions de pesticides, plusieurs techniques sont disponibles :

- partage liquide - liquide entre deux solvants non miscibles,

- chromatographie sur colonne en phase liquide : utilisation de colonne peu sélective, mais à forte capacité. L'adsorbant le plus couramment employé est le «florisil » qui est un silicate de magnésium.

\section{4) Dosages qualitatifs et quantitatifs}

Le fractionnement des pesticides chlorés en mélange extraits, concentrés et purifiés est effectué par chromatographie en phase gazeuse, selon la technique de partage gaz-liquide.

Le dosage est assuré par l'utilisation de détecteur à « capture d'électrons " fonctionnant avec des sources radioactives au tritium ou au nickel 63. Ces appareils sont très sensibles aux pesticides chlorés électrophiles, le minimum de détection étant voisin du picogramme.
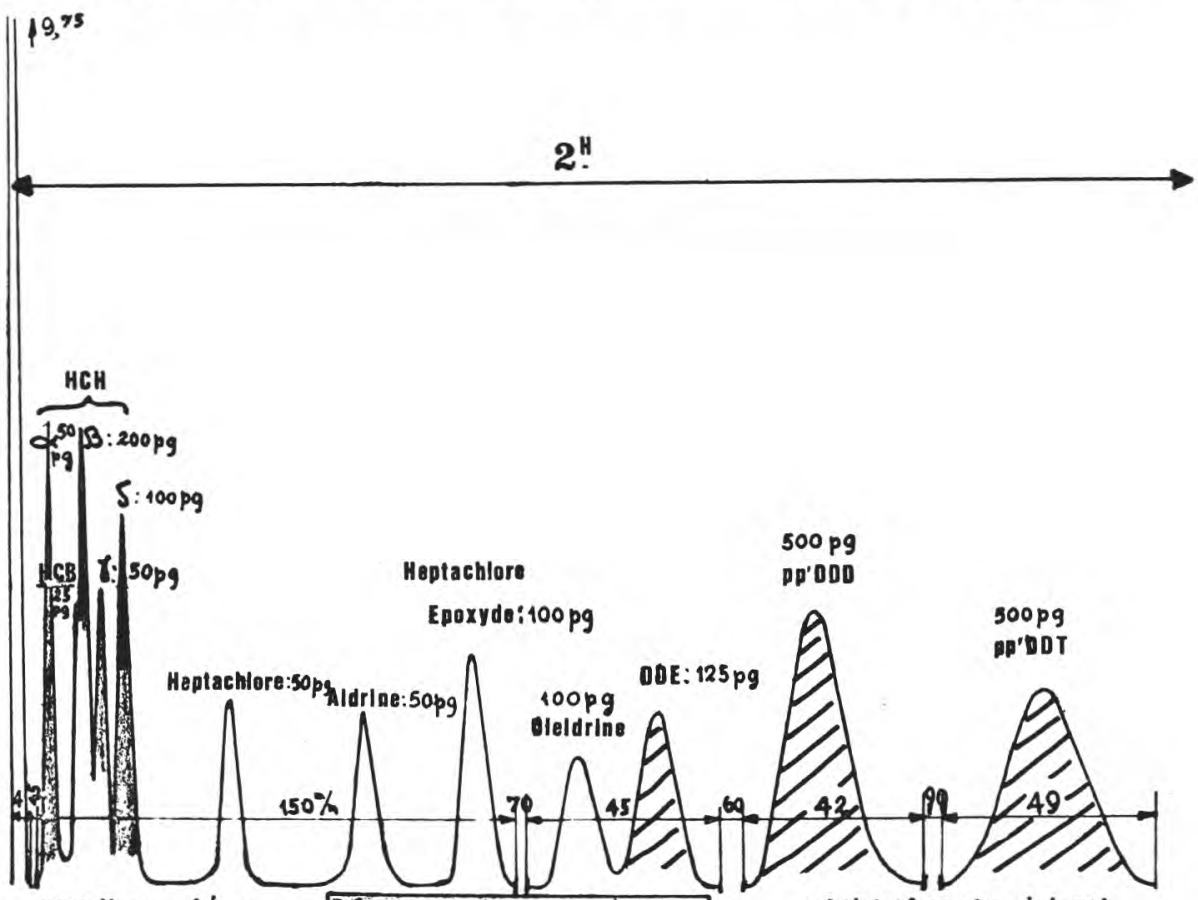

1740 Karian Aérograph DC 200.5\%.Gas-Chrom Q 80/100 Pyrex $10^{\circ} \% / \mathrm{s}^{\circ}+{ }^{\circ} 185^{\circ} \mathrm{C}$ inj: gr/

fig. 7

(chromatogramme 2) 
L'utilisation lors de chaque détermination, de deux ou trois colonnes de polarités différentes permet, en éliminant des pics interférentiels toujours possibles, d'assurer avec justesse les résultats.

Des techniques plus détaillées à ce sujet ont été publiées.

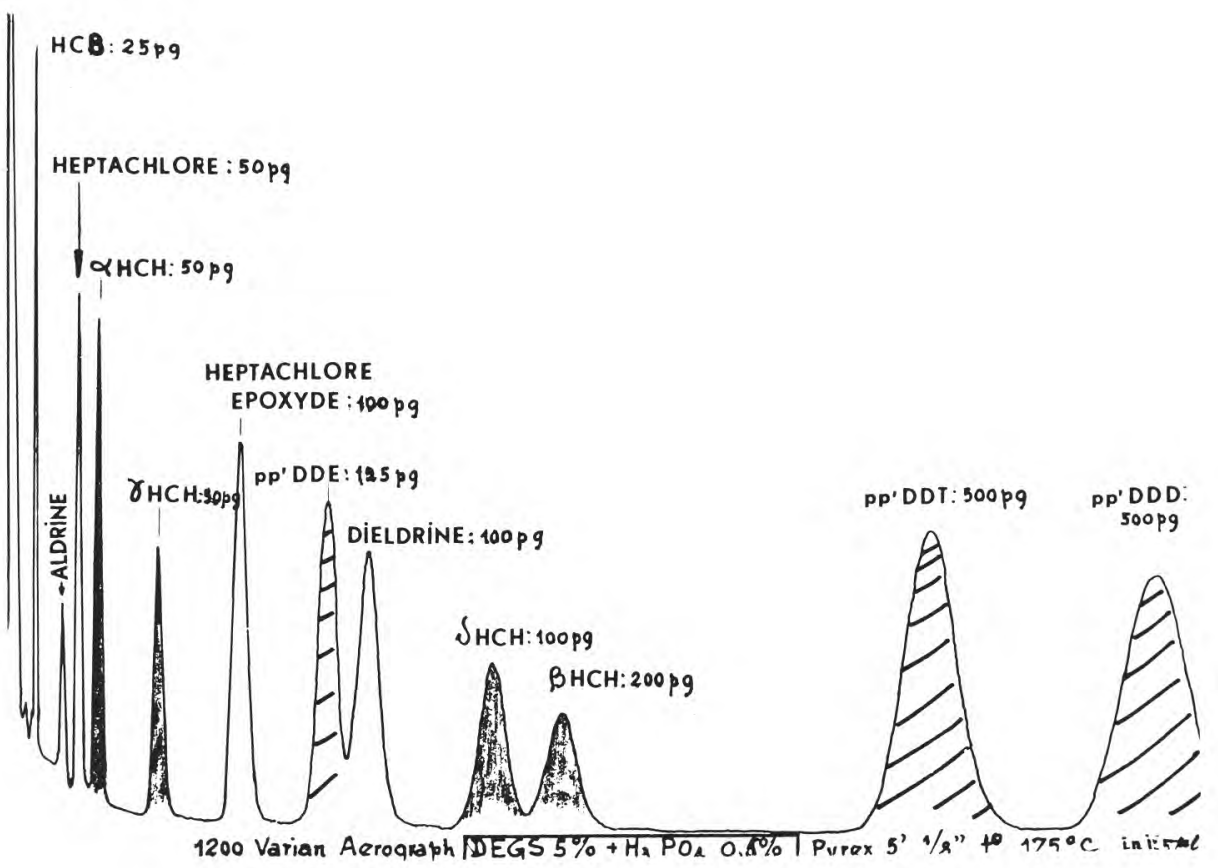

fig. 8

(chromatogramme 3)

\section{RESULTATS}

\section{I. - PRODUITS LAITIERS DE VACHE}

A) Beurre

\section{1) Beurre - enquête de 1967-1968}

Une première enquête a été effectuée, d'octobre 1967 à décembre 1968, sur des beurres pasteurisés de dix-huit beurreries françaises, et en prélevant un échantillon des fabrications de chaque usine, tous les $45 \mathrm{j}$.

Seuls des dosages de $\mathrm{HCH} \alpha$ ont été effectués. Les conclusions de cette étude sont les suivantes :

- les taux de pollution varient largement d'une région à l'autre et aussi d'une usine à l'autre, 
- certaines usines, implantées dans l'Ouest de la France en particulier, présentent une faible pollution tout au long de l'année : 0 à 0,20 ,

- d'autres par contre (Sud-Est et Nord de la France) peuvent être fortement contaminées. Le taux de pollution maximum $(3,5)$ a été observé dans les beurres d'une usine du Nord,

- la pollution est généralement variable au cours de l'année, le maximum se situant en hiver et au printemps, le minimum étant très marqué en été,

tableau 4 .

- l'étude du rapport $\frac{\text { pollution été }}{\text { pollution hiver }}$ est mentionné dans le

\section{2) Beurre : enquête de 1971-1972}

Une enquête identique à celle de 1967-1968 a été effectuée en 1971-1972. Mais les recherches ont porté sur la somme des isomères $\alpha, \beta, \gamma$, et non plus sur le $\mathrm{HCH} \alpha$ seul.

Les conclusions de cette deuxième enquête se résument ainsi :

- les variations comme dans l'enquête précédente sont constatées d'une région à l'autre, et d'une saison à l'autre,

- les régions les plus polluées en $\mathrm{HCH}$ avec des valeurs égales ou supérieures à 0,30 sont le Nord (fig. 10), le Sud-Est et l'Est de la France.

- les maximum de pollution ont été considérablement réduits en 4 ans, et on note une diminution de 80 p. 100 du taux de pollution, par rapport à l'enquête 1967-1968,

- les rapports $\frac{\text { pollution été }}{\text { pollution hiver }}$ sont consignés au tableau 4 ci-après,

- la pollution par $\mathrm{HCH}$ a très fortement diminué d'autant plus qu'il s'agit de la somme des 3 isomères et non plus du seul $\mathrm{HCH} \alpha$; mais on a constaté la présence d'autres pesticides:

- HCB avec des valeurs égales ou supérieures à 0,10 dans le Nord, l'Est, l'Ouest,

- Heptachlore époxyde avec des valeurs égales ou supérieures à 0,20 dans le Nord, l'Est, l'Ouest,

- DDT et dérivés avec des valeurs égales ou supérieures à 0,10 dans l'Ouest, l'Est et le Sud-Est. 
TABLEAU 4. - Pollution des beurres français

Comparaison des rapports de pollution été/hiver

\begin{tabular}{c|c|c|c|c|}
\hline & Nord & Normandie & Ouest & Est \\
\hline 1967 Sud-Est & $1 / 5$ & $1 / 3$ & $1 / 2$ \\
1968 & $1 / 5$ & $1 / 5$ & $1 / 1,5$ \\
1972 & $1 / 3$ & $1 / 1,5$ & $1 / 5$ \\
\hline
\end{tabular}

Comparaison teneur $\mathrm{HCH}$ total phase $72 /$ phase 68

\begin{tabular}{l|c|c|c|c}
\hline & Nord & Normandie & Ouest & Est \\
\hline Hiver & $1 / 5$ & $1 / 3$ & $1 / 4$ & $1 / 3$ \\
Printemps & $1 / 3$ & $1 / 3$ & $1 / 2,5$ & $1 / 2$ \\
Eté & $1 / 7$ & $1 / 4$ & $1 / 5$ & $1 / 3$ \\
Automne & $1 / 4$ & $1 / 8$ & $1 / 4$ & $1 / 4$ \\
\end{tabular}

N.B. - Les résultats concernant les produits laitiers sont exprimés en mg/kg de MG pure. 


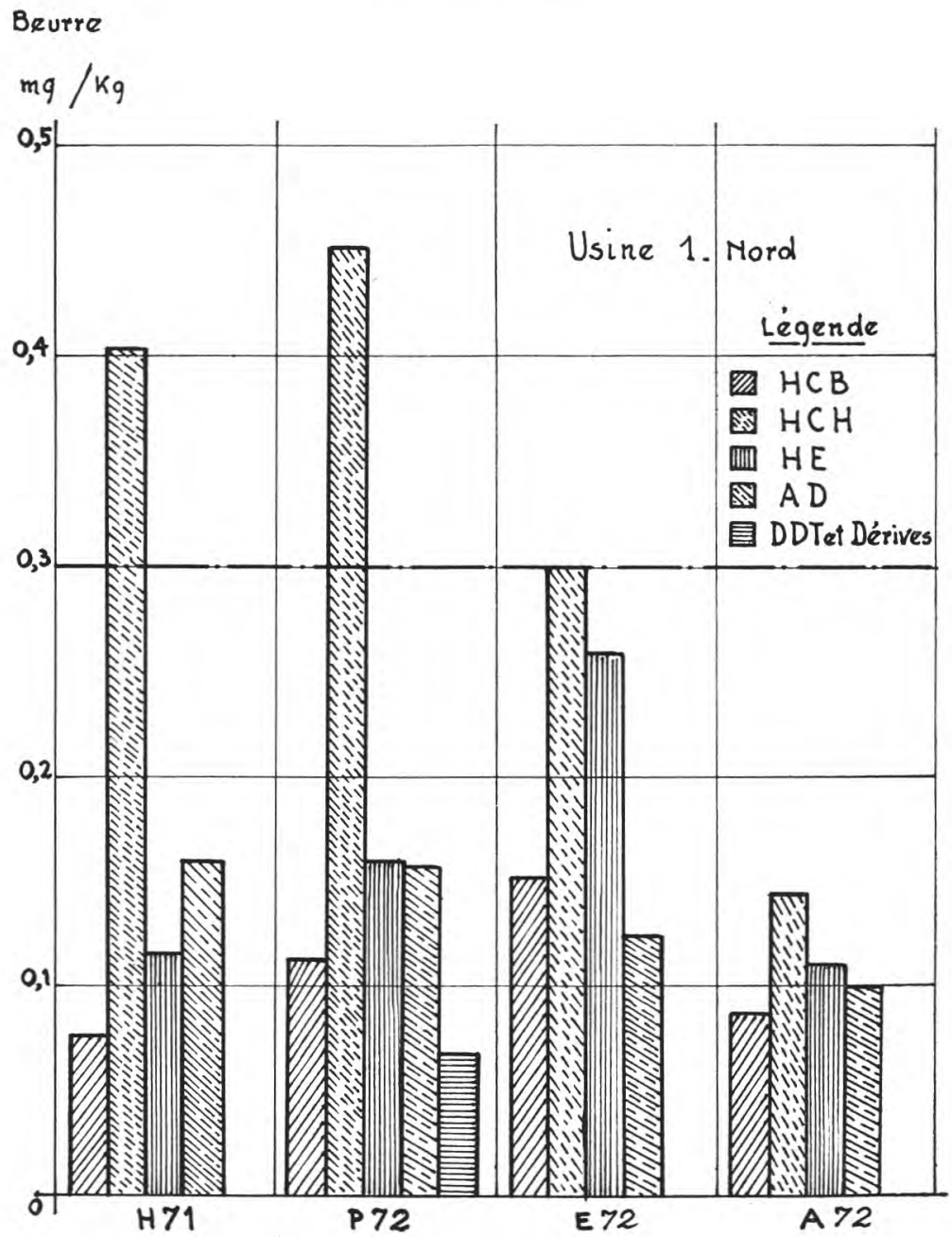

fig. 9

Résidus de pesticides organochlorés. Evolution selon les saisons (années 1971-1972)

\section{3) Conclusions générales des enquêtes beurre}

- en 1967-68

- en 1971-72

- en 1968
85 p. 100 des échantillons ont un taux de pollution égal ou supérieur à 0,20 en $\mathrm{HCH} \alpha$ seul,

35 p. 100 des échantillons ont un taux de pollution égal ou supérieur à 0,20 en $\mathrm{HCH} \alpha+\beta+\gamma$,

le taux moyen de pollution est supérieur à 0,30 sauf dans l'Ouest, 
- en 1972

le taux moyen de pollution est inférieur à 0,10 dans le Nord, la région Rhône-Alpes et la Normandie-Bretagne.

B) Produits LaItiers groupés : LAIts + FROMAgES EN 1972

En 1972, le laboratoire d'Industrie Laitière de Douai a effectué des dosages de résidus de pesticides sur 3000 échantillons de lait de vache, de produits laitiers fabriqués à partir de lait de vache. Les moyennes pondérées des taux de pollution effectuées sur l'ensemble de ces échantillons, non sélectionnés, mais représentatifs des grandes régions laitières françaises, donnent les indications suivantes :

\section{1) Pollution par pesticides}

- pollution par $\mathrm{HCH}$ : dans toutes les régions, mais à des taux différents :

$\geqslant 0,10$ dans le Nord, le Sud-Est et le Sud-Ouest,

$\leqslant 0,10$ en Normandie, dans l'Ouest, la région parisienne, le Centre,

- pollution par heptachlore époxyde :

$\geqslant 0,30$ dans le Nord, la région parisienne,

$>0,10$ dans l'Est et le Sud-Est,

$<$ 0,10 en Normandie, dans l'Ouest, le Sud-Ouest, le Centre,

- pollution par dieldrine :

en général $<0,10$,

- pollution par DDT et ses dérivés :

en général $<0,10$ sauf pour quelques rares cas dans le SudOuest et le Centre,

- les principaux polluants par ordre décroissant d'importance et de fréquence de présence sont :

$\mathrm{HCH}$ sous les formes $\alpha, \beta, \gamma$,

Heptachlore époxyde,

Dieldrine,

DDT et dérivés.

\section{2) Comparaisons des pollutions entre 1969 et 1972}

- maximum de pollution :
en $1969: 0,85$
en Automne,
en $1970: 0,89$
au Printemps,
en $1971: 0,40$
au Printernps,
en $1972: 0,40$
au Printemps, 
- les taux maximum en 1969 et 1970 sont identiques, mais le nombre d'échantillons très pollués en 1970 est réduit de 50 p. 100,

- en 1971, un grand nombre d'échantillons sont pollués mais à un taux très faible,

- en 1972, la diminution de la pollution s'accentue en ce qui concerne le $\mathrm{HCH}$ avec des valeurs moyennes inférieures à 0,20 ,

- l'étude des rapports $\frac{\text { pollution } 1972}{\text { pollution } 1969}$ par région est :

\begin{tabular}{l|l|l|l}
\hline Ouest 1/2 & Nord 1/3 & Est 1/5 & Sud-Est 1/10 \\
\hline
\end{tabular}

\section{II. - PRODUITS LAITIERS DE BREBIS}

\section{1) Le Rayon ou zone de Roquefort}

Taux moyen de pollution pour $\mathrm{HCH} 0,20$; maximum à 1,40.

Les pollutions par heptachlore-époxyde, dieldrine et DDT sont très faibles, en moyenne inférieures à 0,05 .

\section{2) La Corse}

En 1972, pollution en $\mathrm{HCH}$ voisine de 0,10.

\section{3) Les Pyrénées}

Région très polluée avec des maximum en $\mathrm{HCH}$ de 1,00.

\section{III. - PRODUITS LAITIERS DE CHEVRE}

Deux zones de production ont été étudiées.

\section{1) Zone Ouest}

Les échantillons de fromages de chèvre provenant de la Zone Ouest ont été prélevés dans le commerce pendant une année.

Le HCB est souvent présent et varie de 0,08 à 0,12 .

Le $\mathrm{HCH}$ est en moyenne voisin de 0,20 avec quelques maximum à 0,48 en hiver 1971. L'isomère prédominant est le $\gamma \mathrm{HCB}$.

L'heptachlore époxyde est très faible $\leqslant 0,05$.

Le DDT (et dérivés) présente parfois des valeurs élevées jusqu'à 0,19 .

\section{2) Zone Sud-Est}

Des laits de fermes ont été analysés périodiquement.

Le $\mathrm{HCH}$ présente une pollution faible : 0,13.

L'heptachlore époxyde et la dieldrine sont très peu abondants $\leqslant 0,05$ quelques cas isolés de pollution sont à noter $0,46 \mathrm{de} \alpha \mathrm{HCH}$ et $0,23 \beta \mathrm{HCH}$. 


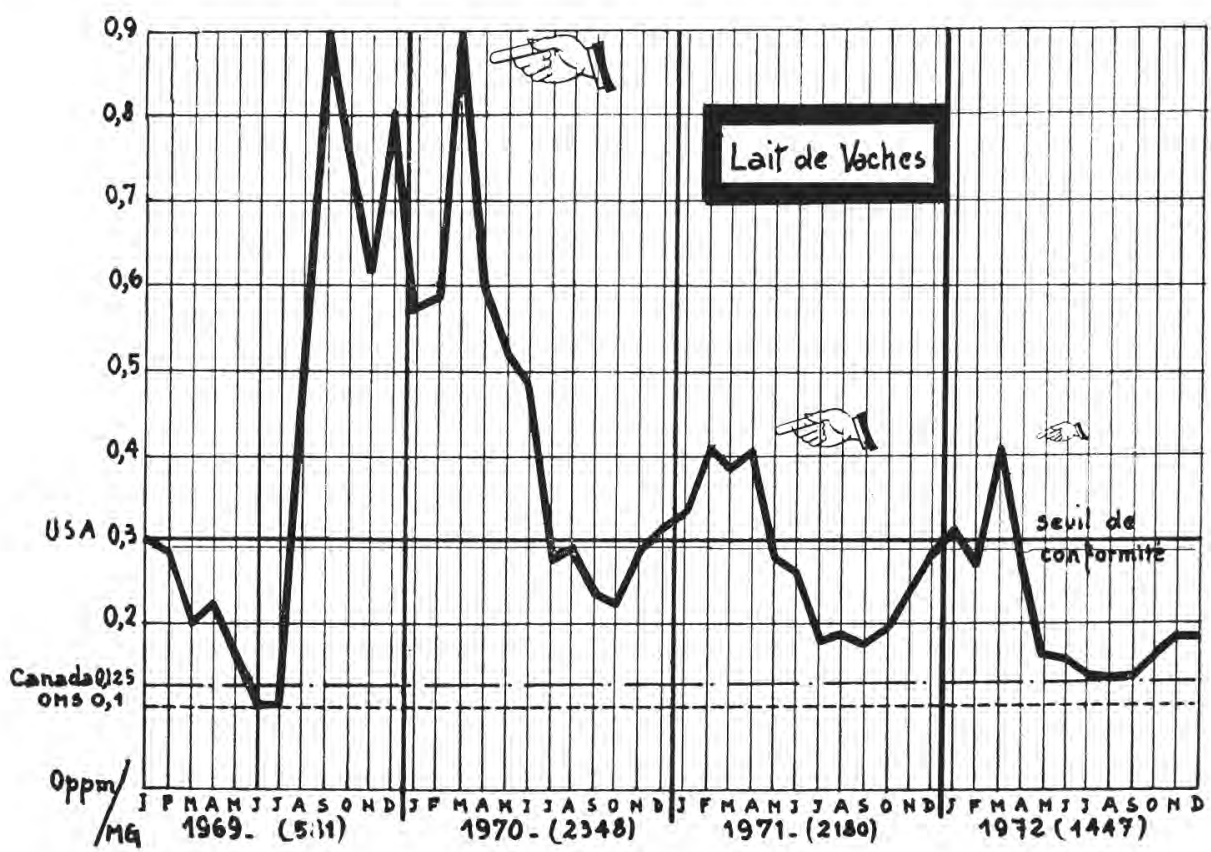

fig. 10

Evolution des résidus de HCH (1969 à 1972)

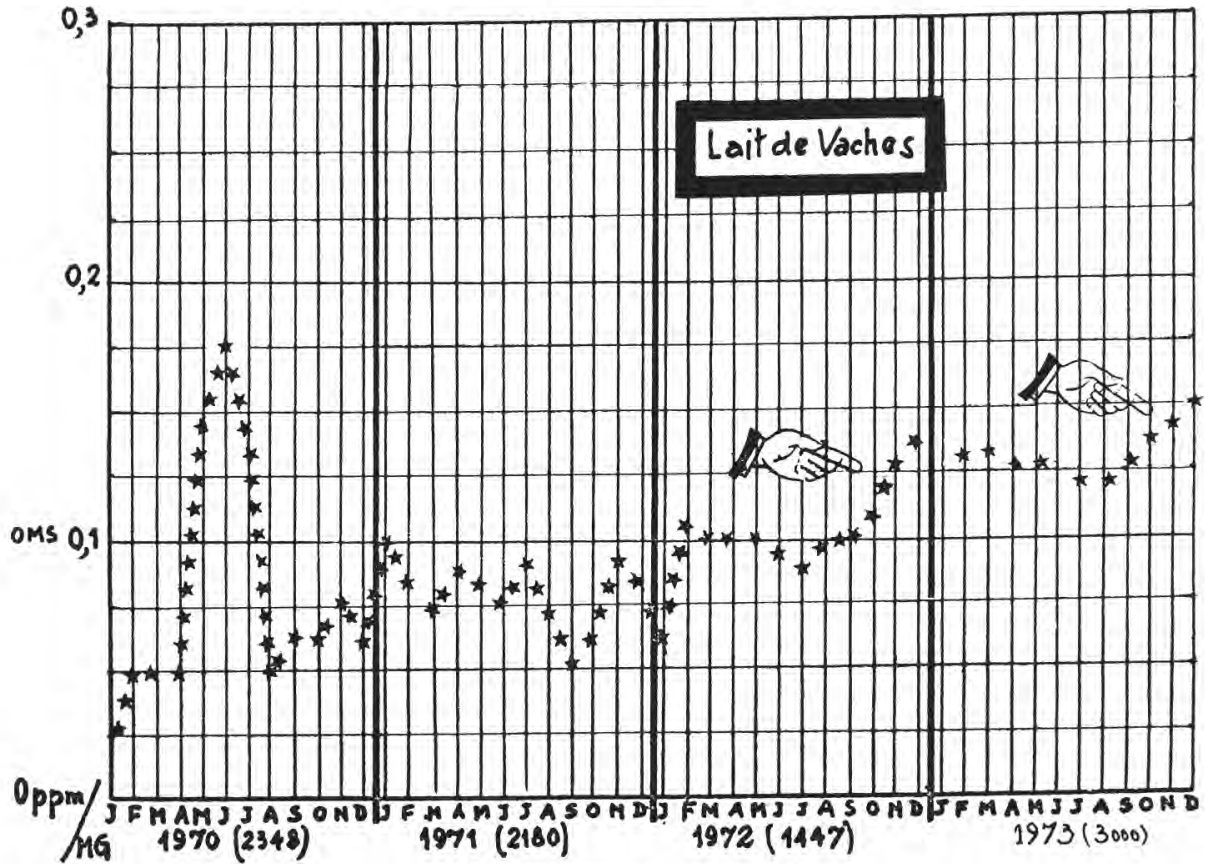

fig. 11

Evolution des résidus de HE (1970 à 1972) 


\section{IV. - BILANS DE POLLUTION (tab. 5)}

\section{1) Lait de vache}

Depuis 5 ans la pollution par $\mathrm{HCH}$ suit une courbe décroissante (fig. 11). Il semble que partout, sauf toutefois partiellement dans le Nord, la tolérance de 0,30 ne soit pas dépassée. Cependant les taux de pollution par l'heptachlore époxyde et la dieldrine suivent une courbe faiblement croissante (fig. 12).

Il faut signaler que de nouvelles difficultés pourraient se présenter, car les tolérances actuelles pourraient être ramenées à un taux plus bas par des réglementations plus sévères, en particulier par celles préconisées par la Communauté Economique Européenne.

\section{2) Lait de brebis}

Le polluant principal est l'HCH, dont le taux de pollution moyen est faible : 0,12. La pollution est en régression, mais avec des variations de faible amplitude.

Les pesticides du type heptachlore époxyde et dieldrine sont apparus, sans créer de difficulté au regard des tolérances en vigueur, mais le seul fait de leur présence, mérite une attention particulière.

\section{3) Lait de chèvre}

Le polluant principal est aussi l' $\mathrm{HCH}$, mais avec une moyenne peu élevée, 0,15 .

Quelques cas de pollution particulière par $\mathrm{HCH} \beta$ ou $\gamma$, et par le DDT ne représentent que des faits isolés et ne sont pas significatifs.

Cependant les reports de lait sous forme de lait sec ou de caillé congelé, permettent un déplacement de la pollution dans le temps.

\section{V. - VOIES ET ORIGINES DE LA POLLUTION}

\section{1) Schéma de pollution du lait (page 291)}

\section{2) Blanchiment des étables}

Avant 1969, on employait fréquemment du $\mathrm{HCH}$ technique ou de l'heptachlore ou de l'aldrine pour désinsectiser les locaux d'élevage.

Un arrêté du 15 octobre 1969 a interdit l'emploi de pesticides organochlorés (DDT, aldrine, dieldrine, $\mathrm{HCH}$ ), pour la désinsectisation des locaux d'élevage.

En 1969, un traitement de blanchiment avec un produit à base de $\mathrm{HCH}$ fait passer de 0,30 à 1,05 , la pollution du lait de l'étable 
TABLEAU 5

Comparaison en 1972 des zones de producteurs de lait d'origine animale

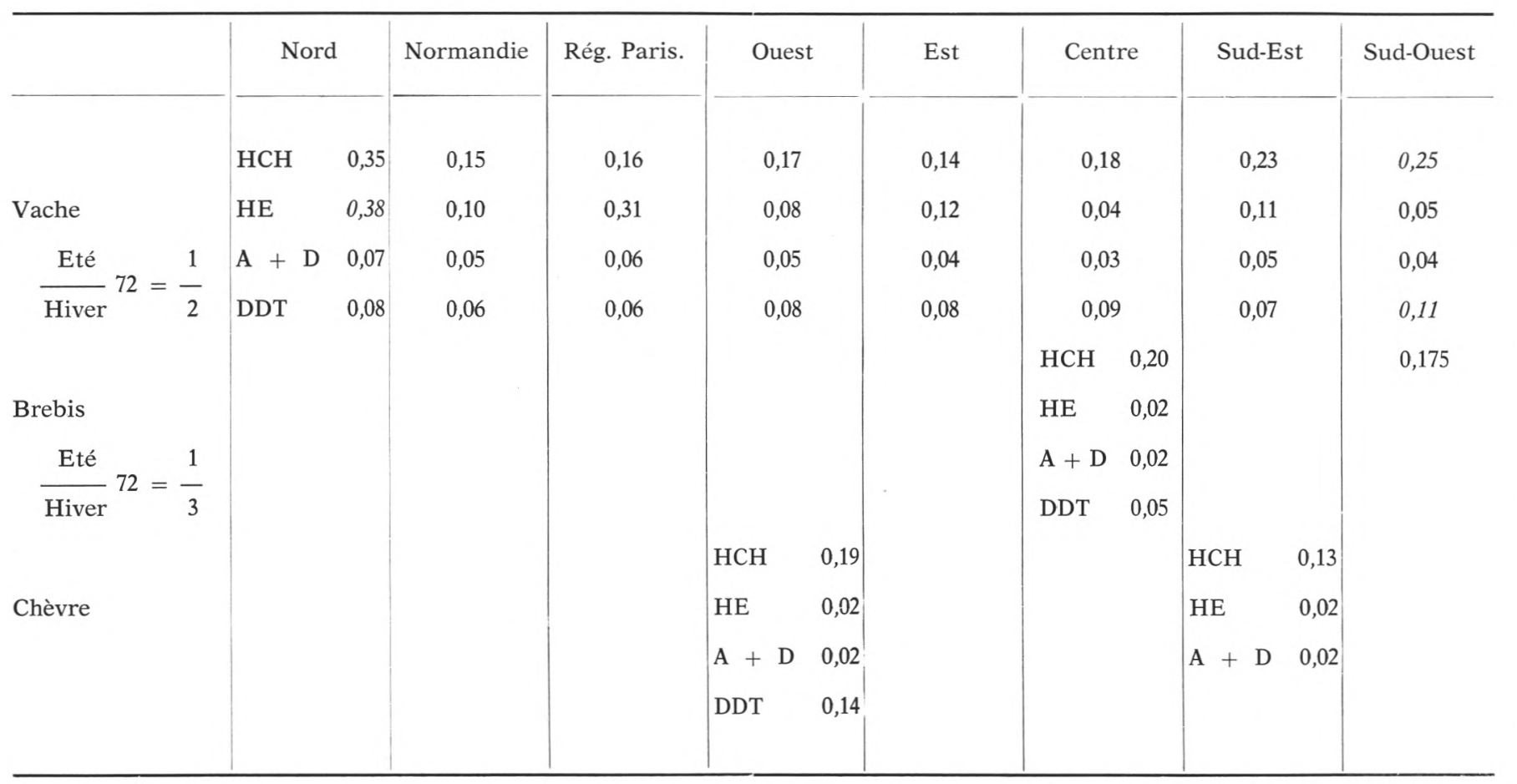


Pollution des produits laitiers par pesticides et insecticides

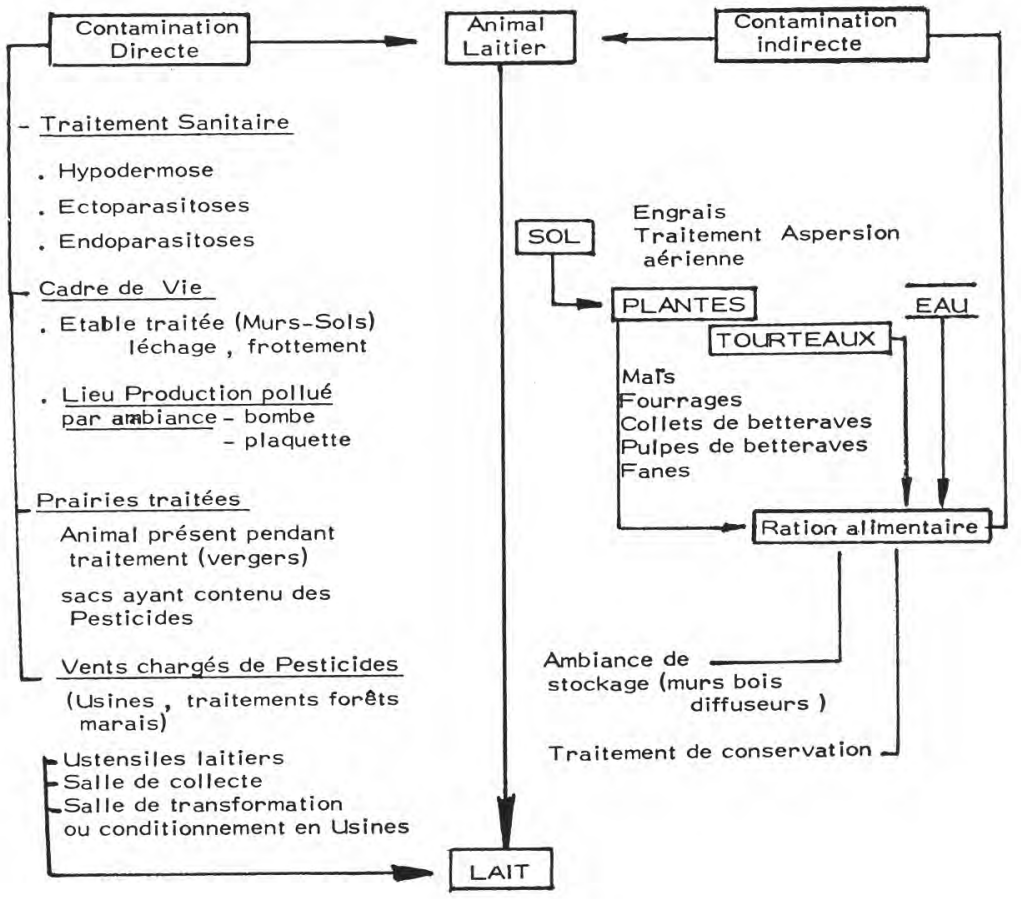

traitée. Encore faut-il remarquer que cette valeur est faible, car le lait analysé a été prélevé longtemps après la rentrée du troupeau à l'étable.

Par contre, en 1970 le traitement de désinsectisation d'une étable au moyen de produits ne contenant pas d'organochlorés, n'a aucune influence sur la pollution du lait qui reste faible avant et après traitement.

\section{3) Traitements vétérinaires}

Effectués lors de certaines parasitoses animales, les produits vétérinaires (hypodermose bovine ou varron, ectoparasitoses dues aux insectes et acariens, endoparasitoses) peuvent contenir des pesticides.

Une expérimentation de traitement externe d'un animal au "lindamonium », produit contenant 30 p. $100 \mathrm{de} \mathrm{HCH} \gamma$, a conduit aux résultats suivants : 
- avant traitement le lait contenait en $\mathrm{HCH} \begin{cases}\alpha & 0,08 \\ \beta & 0,08 \\ \gamma & 0,08\end{cases}$

- après $1 \mathrm{j}: \quad \mathrm{HCH} \gamma=16,6$

$2 \mathrm{j}: \quad \mathrm{HCH} \gamma=6,5$

$4 \mathrm{j}: \quad \mathrm{HCH} \gamma=1,4$

La pollution du lait est donc très rapide et importante, mais elle décroît aussi très brusquement.

En 1971, le contrôle d'un troupeau de brebis, baigné dans une solution contenant du $\mathrm{HCH} \gamma$ au 1/400 a permis de constater la pollution suivante dans le lait :

$$
\begin{array}{rrr}
-24 \mathrm{~h} \text { après : } & \mathrm{HCH} \gamma 40 \\
\alpha & 25 \\
-7 \mathrm{j} \text { après : } & \mathrm{HCH} \gamma & 2 \\
\alpha & 1,8
\end{array}
$$

\section{4) Contamination indirecte}

La ration alimentaire est l'un des facteurs primordial contribuant à polluer le lait. L'alimentation du cheptel peut se trouver contaminée de deux façons :

- pollution primaire : par traitement direct du sol ou du végétal

- pollution secondaire : par traitement lors du stockage ou du transport.

On trouvera dans le tableau 6, un résumé d'enquête de la pollution des aliments et dans le tableau 7 la preuve expérimentale du passage des pesticides de l'aliment pollué dans le lait.

\section{5) Mesures à prendre pour diminuer la pollution}

a) Mise en place d'une législation visant à interdire tous les organochlorés. Ces mesures sont actuellement prises.

b) Contrôler les aliments du cheptel laitier et ne tolérer que ceux dont la pollution répond à des critères très stricts ne permettant pas de contaminer le lait.

c) Promouvoir la recherche de produits de remplacement efficaces, ne risquant pas de laisser des résidus dans le lait. 
TABLEAU 6. - Enquête pollution des aliments donnés aux vaches laitières

\begin{tabular}{|c|c|c|c|c|c|}
\hline Produits & $\begin{array}{c}\text { Nbre échantillons } \\
\text { analysés }\end{array}$ & $\begin{array}{l}\text { Nbre échantillons } \\
\text { non pollués }\end{array}$ & $\begin{array}{c}\text { Nbre échantillons } \\
\text { pollués }\end{array}$ & Polluant & $\begin{array}{l}\text { Taux de pollution } \\
\text { en ppm }\end{array}$ \\
\hline Foin & 89 & 0 & $\begin{array}{rr}89 \\
\text { dont } 40 \\
19 \\
30\end{array}$ & $\begin{array}{l}\mathrm{HCH} \alpha \\
\mathrm{HCH} \alpha \\
\mathrm{HCH} \alpha\end{array}$ & $\begin{array}{c}0,01 \\
\text { de } 0,01 \text { à } 0,02 \\
>0,02\end{array}$ \\
\hline Céréales & 71 & 40 & $\begin{array}{cc}31 \\
\text { dont } 27 \\
11\end{array}$ & $\begin{array}{l}\mathrm{HCH} \alpha \\
\mathrm{HCH} \text { et autres } \\
\text { pesticides }\end{array}$ & 0,030 à 6,06 \\
\hline Choux et colza & 20 & 4 & $\begin{array}{rr}16 & \\
\text { dont } & 9 \\
& 2 \\
& 5\end{array}$ & $\begin{array}{l}\mathrm{HCH} \\
\text { Heptachlore époxyde } \\
\text { Dieldrine }\end{array}$ & $\begin{array}{l}0,01 \\
0,01 \\
0,01\end{array}$ \\
\hline Betteraves & 33 & 17 & 16 & $\begin{array}{l}\text { Heptachlore époxyde } \\
\text { Dieldrine }\end{array}$ & $\begin{array}{l}0,01 \text { à } 0,45 \\
0,03 \text { à } 0,16\end{array}$ \\
\hline Pulpes fraîches & & & & Heptachlore & $\begin{array}{c}0,01 \text { à } 0,45 \\
\text { dans la M.S. }\end{array}$ \\
\hline Maïs grain & 7 & 0 & 7 & $\mathrm{HCH}$ & 0,025 à 0,065 \\
\hline Tourteaux d'arachide & 22 & 9 & $\begin{array}{r}13 \\
\text { dont } 12 \\
8\end{array}$ & $\begin{array}{l}\mathrm{HCH} \alpha \\
\mathrm{HCH} \beta\end{array}$ & de 0,03 à 0,48 \\
\hline Ensilage maïs & 26 & 15 & $\begin{array}{rr}11 & \\
\text { dont } & 6 \\
& 5\end{array}$ & $\begin{array}{l}\text { HCH } \alpha \text { et } \gamma \\
\text { Heptachlore époxyde }\end{array}$ & 0,01 \\
\hline Ensilage divers & 11 & 3 & $\begin{array}{rr}8 & \\
\text { dont } & 6 \\
& 2\end{array}$ & $\begin{array}{l}\mathrm{HCH} \alpha \\
\text { Heptachlore époxyde }\end{array}$ & 0,01 \\
\hline
\end{tabular}


Incidence de la pollution des aliments sur celle du lait (constatation expérimentale)

\section{TABLEAU 7}

Constatation du passage des pesticides des aliments dans le lait après distribution journalière pendant 2 à 3 mois

\begin{tabular}{|c|c|c|c|c|c|}
\hline \multirow{2}{*}{$\begin{array}{l}\text { Pesticides } \\
\text { polluants }\end{array}$} & \multirow{2}{*}{\multicolumn{2}{|c|}{ Pollution aliments* }} & \multicolumn{3}{|c|}{ Pollution du lait** } \\
\hline & & & P1 & P2 & $\mathrm{P} 3$ \\
\hline \multirow[t]{4}{*}{$\mathrm{HCH}$} & Concentré & 0,25 & 0,93 & 0,41 & 0,25 \\
\hline & Tourteaux & 1,75 & 0,92 & 1,50 & 0,16 \\
\hline & Céréales et choux & 0,11 & 0,60 & 1,30 & 0,60 \\
\hline & Céréales & 12,80 & 10,90 & 4,60 & 0,60 \\
\hline \multirow[t]{3}{*}{ Heptachlore } & Racines de betteraves & 0,25 & 0,27 & 0,42 & 0,41 \\
\hline & Pulpes de betteraves & 0,46 & 1,18 & 1,10 & 0,50 \\
\hline & Ensilage maïs & 0,034 & 0,18 & 0,32 & 0,24 \\
\hline \multirow[t]{3}{*}{ Aldrine et dieldrine } & Foin & 0,034 & 0,10 & 0,35 & 0,28 \\
\hline & Pulpes de betteraves & 0,025 & 0,05 & 0,12 & 0,14 \\
\hline & Tourteaux & 0,015 & 0,11 & 0,11 & 0,09 \\
\hline
\end{tabular}

* mg de pesticides par rapport à la matière sèche totale de l'aliment.

** mg de pesticides par rapport à la matière grasse pure.

L'aptitude des pesticides contenus dans les aliments à polluer le lait est la suivante, par ordre décroissant :

heptachlore époxyde - dieldrine - $\mathrm{HCH} \alpha-\mathrm{HCH} \gamma$

\section{VI. - LAITS DE FEMME}

Deux enquêtes ont été effectuées par le Laboratoire d'Industrie Laitière de Douai, l'une sur le plan régional, l'autre sur le plan national, au moyen d'échantillons de lait en provenance de différents lactariums.

\section{1) Enquête régionale}

De 1970, sur des laits en provenance des lactariums des départements du Nord et du Pas-de-Calais. 
Les résultats de cette enquête sont donnés dans le tableau 8 . Ils sont exprimés en $\mathrm{mg}$ par $\mathrm{kg}$ de matière grasse pure.

\section{TABLEAU 8}

\begin{tabular}{l|c|c|c}
\hline \multicolumn{1}{c|}{ Pesticides } & Moyenne & Minimum & Maximum \\
\cline { 1 - 2 } & & & \\
$\alpha+\beta+\gamma$ & 2,64 & 0,6 & 6,06 \\
dont $\beta$ seul & 2,49 & 1,05 & 6,00 \\
HCB & 1,50 & 0,30 & 5,10 \\
Heptachlore époxyde & 0,25 & 0,12 & 0,48 \\
Dieldrine & 0,39 & 0,01 & 0,96 \\
& & & \\
\hline
\end{tabular}

\section{2) Enquête nationale}

Effectuée sur des échantillons de lait provenant de dix lactariums suivants : Lille 1 et Lille 2, Rouen, Paris, Strasbourg, Nantes, SaintEtienne, Lyon, Bordeaux, Marmande qui ont fourni chaque mois et pendant une année, des échantillons.

a) Résultats de l'enquête

- La pollution moyenne des laits de femme est importante :

Pollution par $\mathrm{HCH}+\mathrm{HCB}: 2,75$ soit 5 à 10 fois plus que la pollution des laits de vache.

Pollution par heptachlore époxyde : 0,28 soit 4 à 5 fois plus que la pollution des laits de vache.

Pollution par dieldrine : 0,23 soit 5 à 6 fois plus que la pollution des laits de vache.

Pollution par DDT et dérivés : 3,24 soit 50 à 100 fois plus que la pollution des laits de vache.

- Les polluants les plus caractéristiques des laits de femme sont les suivants :
HCB
$\mathrm{HCH} \beta$
Dérivés du DDT

$\begin{array}{ll}\text { moyenne nationale } & 0,98 \\ \text { moyenne nationale } & 1,67 \\ \text { moyenne nationale } & 3,24\end{array}$

A l'inverse de ce qui a été constaté pour les laits de vache, de brebis ou de chèvre, la pollution des laits de femme ne présente pas de variation saisonnière.

Tous les échantillons sont pollués, cependant à des degrés divers. 
- Taux de pollution les plus élevés :

\begin{tabular}{|c|c|}
\hline $\begin{array}{l}\text { Pollution par } \mathrm{HCH} \text { : } \\
2,1 \text { de moyenne, au Sud de la Loire }\end{array}$ & $\begin{array}{l}\text { Lyon } \\
\text { Nantes } \\
\text { St-Etienne }\end{array}$ \\
\hline $\begin{array}{l}\text { Pollution par heptachlore époxyde et } \\
\text { dieldrine : } \\
0,70 \text { de moyenne, au Nord de la Seine }\end{array}$ & $\begin{array}{l}\text { Lille } \\
\text { Rouen } \\
\text { Paris }\end{array}$ \\
\hline $\begin{array}{l}\text { Pollution par DDT, sur l'ensemble } \\
\text { des échantillons dont en particulier }\end{array}$ & $\begin{array}{l}\text { Paris } \\
\text { Marmande } \\
\text { Lille }\end{array}$ \\
\hline
\end{tabular}

- Taux de pollution les plus faibles :

Pollution par $\mathrm{HCH}$

Pollution par heptachlore époxyde

Pollution par dieldrine

Pollution par DDT et dérivés

$\begin{array}{ll}\text { Strasbourg } & 1,24 \\ \text { Marmande } & 1,46 \\ \text { Bordeaux } & 1,52 \\ \text { St-Etienne } & 0,31 \\ \text { Lyon } & 0,44 \\ \text { Strasbourg } & 2,16\end{array}$

Les taux de pollution des laits de femme sont très supérieurs à ceux qui sont tolérés par la réglementation pour les laits animaux.

Si l'on compare les résultats obtenus sur les échantillons en provenance du Lactarium de l'Institut Pasteur de Lille en 1970 et en 1972 :

$\begin{array}{ccc} & \text { HCH } & \text { Heptachlore époxyde } \\ 1970 & 2,60 & 0,53 \\ 1972 & 1,80 & 0,25\end{array}$

On constate que le taux de pollution a diminué d'environ 50 p. 100 en 2 ans, et cette évolution est à rapprocher de celle observée pour les laits animaux. Cette amélioration est vraisemblablement une conséquence des mesures prises par les Pouvoirs Publics pour limiter ou même interdire totalement l'usage de pesticides organochlorés.

La pollution des laits de femme est certainement influencée par l'origine des mères. Le mode de vie et les habitudes nutritionnelles jouent un rôle prépondérant. C'est ainsi que l'analyse d'échantillons de lait provenant de femmes algériennes a donné les résultats suivants :

- Une forte pollution en HCB

- Une notable pollution en $\mathrm{HCH} \beta$

- Une forte pollution en pp' DDE moyenne 3,27 avec un maximum à 10,9

moyenne 0,94

moyenne 3,54

b) Origines de la pollution des laits de femme:

- Facteur d'ambiance : utilisation ménagère massive de plaquet- 
tes ou bombes insecticides (1 bombe consommée pour 4 habitants en 1972).

\section{- L'alimentation.}

Les aliments constituent un facteur important de pénétration des pesticides dans l'organisme. On constate la présence de résidus de pesticides dans la plupart des denrées alimentaires :

$\mathrm{HCH}$

$\mathrm{HCH}$

Heptachlore époxyde

Dieldrine

HCB

DDT dans les viandes, la charcuterie le lait, les matières grasses 0,01 à 0,30 dans les carottes, le gibier 0,07 à 0,20 viandes, huiles, les produits laitiers.

dans les carottes, les oufs... dans les salades, les endives...

dans les matières grasses des animaux marins.

Pour les laits de femme, les taux de pollution par les résidus de pesticides sont 5 à 10 fois supérieurs à ceux des laits de vache. On peut à ce sujet formuler une hypothèse qui pourrait servir de base à des recherches ultérieures :

Les résidus de pesticides chlorés s'accumulent dans les tissus adipeux. Une faible partie de ces résidus est éliminée par les urines et les fécès, mais la majeure partie est excrétée pendant la sécrétion lactée par l'intermédiaire des globules gras, or :
poids corporel moyen
$1^{\circ}$ En effectuant le rapport PCM

on constate que ce rapport

$\begin{array}{ll}\text { est six fois plus élevé chez la femme } & \frac{60}{40}=1,5 \\ \text { que chez la vache } & \frac{600}{2300}=0,26\end{array}$

$2^{\circ}$ En comparant les conditions de vie de la femme et de la vache on peut faire les constatations suivantes :

Début de la production du lait

$\begin{array}{ll}\text { Femme } & \text { Vache } \\ \text { vers } 20 \text { ans } & \text { vers } 2 \text { ans } \\ 2 \text { à } 3 \text { mois } & 10 \text { mois } \\ 1 \text { à } 3 & 4 \text { à } 6\end{array}$

Durée de la lactation

1 à 3

4 à 6

Ces différences importantes peuvent semble-t-il expliquer pour partie les différences de pollution des laits de femme et des laits de vache par les résidus de pesticides organochlorés. 


\section{CONCLUSIONS}

Les pesticides sont absolument nécessaires actuellement pour maintenir les rendements des productions agricoles et pour lutter contre les maladies épidémiques. On ne peut donc raisonnablement envisager d'en interdire l'emploi.

Certains de ces produits, tels les organochlorés, sont très rémanents et liposolubles. De ce fait ils laissent des résidus tout au long de la chaîne alimentaire, les concentrations en ces produits étant les plus élevées dans les produits riches en lipides.

Il est certain que pour l'homme, ces produits présentent des risques de toxicité, soit sous forme aiguë (fréquence minime), soit sous forme chronique par l'ingestion répétée au cours des années de petites doses de résidus de pesticides. C'est pour cela que l'Organisation Mondiale de la Santé a défini des tolérances en ce qui concerne les doses maxima que les aliments peuvent contenir.

\section{Pollution actuelle des laits en France (fig. 12)}

Lait de vache : La pollution varie toujours suivant les régions, d'une part, suivant les saisons d'autre part avec un maximum en hiver.

De 1969 à 1972 la pollution a diminué dans un rapport de 4 à 1 .

Actuellement la pollution moyenne est la suivante :

$\begin{array}{ll}\mathrm{HCH}+\mathrm{HCB} & 0,20 \\ \text { Heptachlore époxyde } & 0,15 \\ \text { Dieldrine } & 0,05 \\ \text { DDT et dérivés } & 0,08\end{array}$

Cette pollution reste en moyenne, inférieure aux tolérances communément admises. Cependant par régions ou par zones de collecte on notera des écarts à la pollution moyenne. Un seul exemple, concernant le Nord :
$\mathrm{HCH}$
0,35
Heptachlore époxyde 0,38

Une dernière observation sera consacrée à l'heptachlore époxyde, et à la dieldrine, dont les taux, inférieurs aux normes actuelles, n'ont cessé depuis 1969 de croître régulièrement.

Lait de brebis : On observe des phénomènes très semblables à ceux constatés pour les laits de vache. La présence momentanée de $\mathrm{HCH} \gamma$ en grande quantité s'explique par l'utilisation de lindane dans certaines thérapeutiques vétérinaires. Cependant, en 1972, la pollution des laits de brebis est inférieure d'environ 50 p. 100 à celle des laits de vache. 


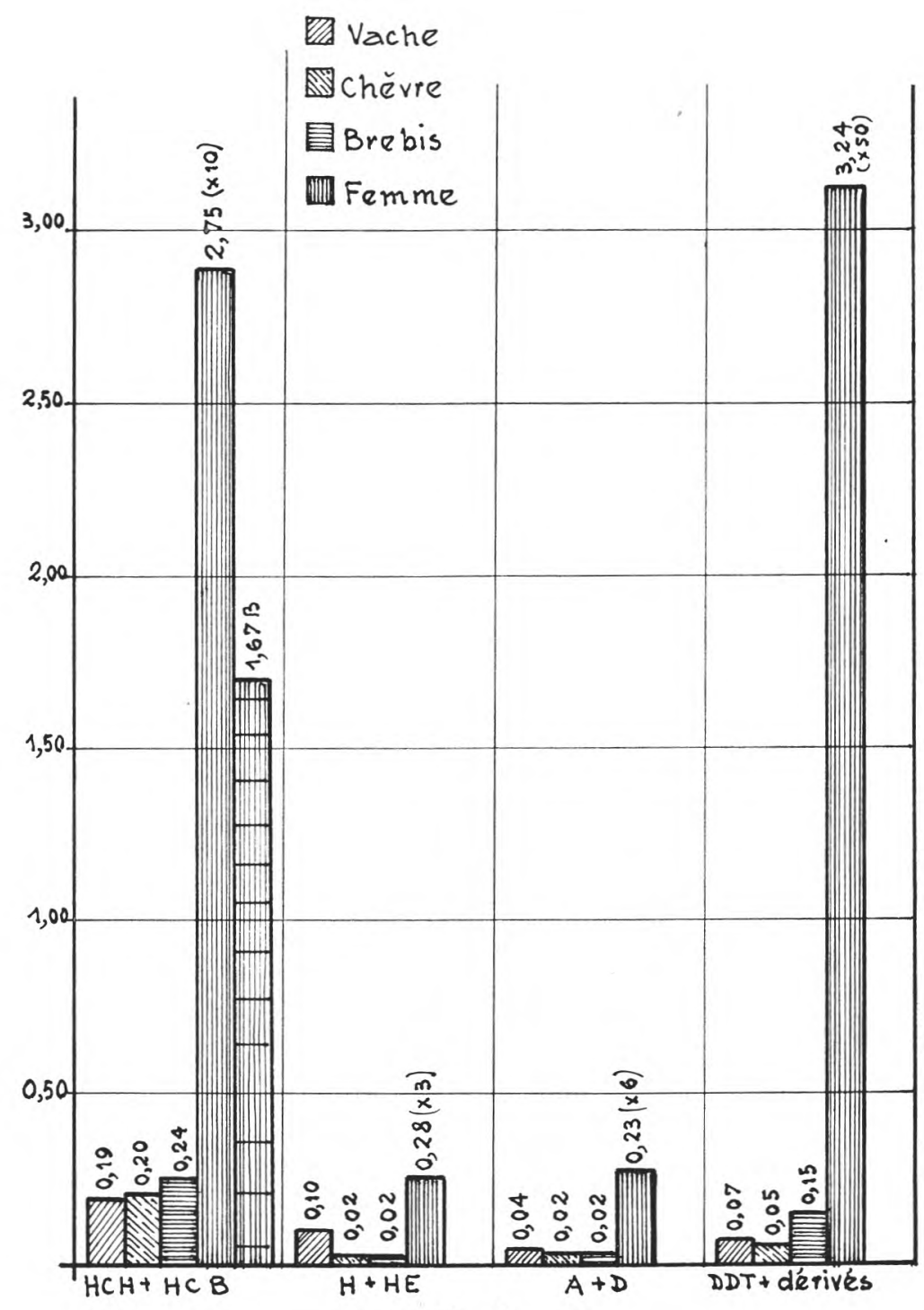

fig. 12

Taux moyen comparé de pollution des différents laits (1971-1972)

Lait de chèvre : La pollution des laits de chèvre est également semblable à celle des laits de vache avec quelques nuances concernant le $\mathrm{HCH} \beta$ et le DDT. Les reports de laits en poudre ou de caillés congelés peuvent déplacer la pollution dans le temps. 
Pour tous les laits animaux on note une très forte diminution des résidus de $\mathrm{HCH}$, et une présence faible mais croissante d'heptachlore époxyde et de dieldrine.

Lait de femme: Les laits de femme sont beaucoup plus pollués que les laits d'animaux. Les polluants majeurs sont le $\mathrm{HCB}$, le $\mathrm{HCH}$ et les dérivés du DDT. Pour ces produits, la pollution se compte en $\mathrm{mg} / \mathrm{kg}$ de MG pure plus qu'en fractions de $\mathrm{mg}$.

\section{Voies de pollution}

Lait animaux : Les laits animaux sont contaminés de manière indirecte, soit par des traitements vétérinaires ou de désinsectisation des locaux, soit par ingestion de nourriture polluée. Certains pesticides ont plus d'aptitude que d'autres à polluer le lait, l'ordre décroissant est le suivant :

$$
\text { Heptachlore époxyde - dieldrine - } \mathrm{HCH} \alpha-\mathrm{HCH} \gamma
$$

Des mesures ont été prises par les Pouvoirs Publics pour réduire la pollution du lait ; en particulier elles ont supprimé totalement l'autorisation d'emploi d'organochlorés en agriculture.

Lait de femme : Les origines de la pollution sont d'une manière générale alimentaire, mais elles varient suivant les milieux sociaux et les habitudes alimentaires.

\section{R és u m é}

Les pesticides faisant l'objet de recherches systématiques dans le lait et les produits laitiers, sont les résidus d'insecticides organochlorés liposolubles.

Les polluants les plus caractéristiques des produits laitiers français sont le $\mathrm{HCH}(\alpha, \beta, \gamma)$; le $\mathrm{HCB}, l^{\prime}$ heptachlore époxyde, la dieldrine.

Les moyennes de pollution sont les suivantes, exprimées en mg par $\mathrm{kg}$ de matière grasse pure : $\mathrm{HCH}$, tous isomères compris 0,20 ; heptachlore époxyde 0,15; dieldrine et $\mathrm{HCB}<0,10$.

Pour les fromages de brebis, le polluant majeur est le $\mathrm{HCH}$ (moyenne 0,12). Les fromages de chèvre présentent une pollution semblable : $\mathrm{HCH}$ taux moyen 0,15 .

Comparativement aux laits animaux, les laits de femmes françaises sont très pollués. Les valeurs moyennes annuelles sont les suivantes : HCH 1,77; HCB 0,98; heptachlore époxyde 0,28 ; dieldrine 0,$23 ; \mathrm{DDT}+$ DDE 3,24.

La pollution des laits et des produits laitiers est principalement due à l'ingestion par les vaches laitières, d'alimentation généralement contaminée. 


\section{S u $\mathbf{m} \mathbf{m}$ a r y}

Pesticides systematically studied in milk and milk products, are the fat soluble organochlorine insecticide residues.

The contaminants characteristic of french milk products are BHC $\alpha, \beta, \gamma), \mathrm{HCB}$; Heptachlor-epoxide, Dieldrin.

The averages of pollution, are, on a fat basis in $\mathrm{mg}$ par $\mathrm{kg}$ : $\mathrm{BHC}$, all isomers 0,20; Heptachlor-epoxide 0,15; Dieldrin and $\mathrm{HCB}<0,10$.

For ewe cheese, the major contaminant is BHC (average 0,12). Goat cheese have a similar pollution : BHC 0,15.

Human milk of French mothers is very high in pesticides compared to animals' milk. Amounts of annual averages are : BHC 1,77 ; HCB 0,98 ; heptachlor-epoxide 0,28 ; dieldrin 0,23 ; DDT and DDE 3,24.

Contaminated animal feed is the principal source from which dairy cattle acquire pesticides, which they eliminate in milk fat.

Reçu pour publication le 26 février 1974.

\section{Bibliographie}

-. Cf. Thèse François-Marie LuQueT, 450 réf.

\section{Remerciement}

Nous tenons à remercier tous ceux qui ont participé à ce travail et particulièrement les Directeurs d'entreprises laitières, les Directeurs des Lactariums et les Responsables d'Organismes interprofessionnels. 